\title{
Ganoderic acid A inhibits ox-LDL-induced THP-1-derived macrophage inflammation and lipid deposition via Notch1/PPAR $/$ CD36 signaling
}

\author{
Tao Wang ${ }^{A-F}$, Huihe Lu ${ }^{A-C, E, F}$ \\ Department of Cardiology, Second Affiliated Hospital of Nantong University, China \\ A - research concept and design; $\mathrm{B}$ - collection and/or assembly of data; $\mathrm{C}$ - data analysis and interpretation; \\ $D$ - writing the article; $E$ - critical revision of the article; $F$ - final approval of the article
}

Address for correspondence

Tao Wang

E-mail:wangtaowtww@163.com

Funding sources

None declared

Conflict of interest

None declared

Received on January 27,2021

Reviewed on April 5, 2021

Accepted on May 20, 2021

Published online on July 30, 2021

Cite as

Wang T, Lu H. Ganoderic acid A inhibits ox-LDL-induced THP-1-derived macrophage inflammation and lipid

deposition via Notch1/PPARY/CD36 signaling.

Adv Clin Exp Med. 2021;30(10):1031-1041.

doi:10.17219/acem/137914

DOI

10.17219/acem/137914

Copyright

Copyright by Author(s)

This is an article distributed under the terms of the

Creative Commons Attribution 3.0 Unported (CC BY 3.0)

(https://creativecommons.org/licenses/by/3.0/)

\begin{abstract}
Background. Atherosclerosis (AS), a chronic inflammatory disease, is a major contributor to deaths worldwide. Ganoderic acid A (GAA) has been widely applied for various diseases due to its excellent antiinflammatory properties.

Objectives. To investigate the underlying mechanism of GAA inhibition of inflammation and lipid deposition in human monocyte (THP-1) cells.

Materials and methods. The Cell Counting Kit-8 (CCK-8) assay was used to assess the potential effect of GAA on the viability of THP-1 cells. The release of inflammatory cytokines and oxidative stress was measured using enzyme-linked immunosorbent assay (ELISA) and the corresponding kit, respectively. The levels of lipid deposition and total cholesterol (TC) were also evaluated. Next, the scavenger receptors and proteins in Notch1/ PPARy/CD36 signaling were measured with western blot. As Notch1 was overexpressed in the THP-1 cells induced by oxidized low-density lipoprotein (ox-LDL), the above assays were performed again to confirm the underlying mechanism.

Results. Ganoderic acid A suppressed ox-LDL-induced inflammation and oxidative stress in THP-1 cells. At the same time, it inhibited the TC level and lipid deposition. The effects of GAA on alleviating inflammation, oxidative stress and lipid accumulation were relieved after the overexpression of Notch1 in the treated cells, and the effects of GAA on alleviating inflammation, oxidative stress and lipid accumulation were diminished. The PPARy activator also weakened the effects of GAA on relieving inflammation, oxidative stress and lipid accumulation in 0x-LDL-induced THP-1 cells.
\end{abstract}

Conclusions. Ganoderic acid A inhibits ox-LDL-induced macrophage inflammation and lipid deposition in THP-1 cells through Notch1/PPARy/CD36 signaling, which may provide theoretical guidance for the clinical applications of GAA in AS treatment.

Key words: inflammation, Notch1, PPARy, ganoderic acid A, lipid deposition 


\section{Background}

Atherosclerosis (AS), a chronic inflammatory disease, is a leading cause of death worldwide. ${ }^{1}$ The loading of cholesterol in macrophages of the artery walls contributes to lipid deposition, which is believed to induce the occurrence and development of AS. ${ }^{2}$ Therefore, AS is considered to have a close relationship with lipid deposition. Apart from lipid deposition and long-term inflammation, immune activation and the stimulation of inflammatory mediators are also AS characteristics. Therapies targeting anti-inflammatory agents are thought to be useful methods for the treatment of AS, but investigation and studies of agents that possess anti-inflammatory effects for the resolution of AS occurrence are limited. Thus, there is a desperate need to study and develop new agents focusing on the inhibition of inflammation for the effective treatment of AS.

Ganoderma lucidum has been widely used in traditional Chinese medicine for the treatment of inflammatory diseases, cancers and immunological diseases. ${ }^{3}$ It has been applied for thousands of years due to its remarkable physiological and pharmacological activities. Ganoderic acid A (GAA) is one of the major triterpenoids in Ganoderma lucidum. Many experts have confirmed that GAA plays an important role in treating cancers because of its efficacy in preventing proliferation and enhancing apoptosis of multiple cancer cells. ${ }^{4,5}$ The anti-inflammatory and antioxidant roles of GAA cannot be neglected because rats injected with GAA have been shown to be protected from liver injury and lung injury induced by various factors. ${ }^{6,7}$ The latest findings show that GAA has great potential to inhibit Notch1 protein expression, which is believed to enhance the stability of peroxisome proliferator-activated receptor gamma (PPAR $\gamma$ ) and increase the level of CD36 in human monocyte (THP-1)-derived macrophages stimulated by interleukin (IL)-4. ${ }^{8,9}$ Lipid deposition in macrophage foam cells can contribute to the formation of plaques and exacerbate the development of AS. Inflammation is a critical risk factor for the occurrence of AS. ${ }^{10}$ Therefore, we speculated that GAA may alleviate the symptoms of AS by inhibiting inflammation and lipid deposition in THP1-derived macrophages via the Notch1 signal.

A large quantity of low-density lipoprotein (LDL) is modified to oxidized low-density lipoprotein (ox-LDL), which aggregates in the vascular internal wall and triggers atherosclerotic plaque development. ${ }^{11}$ Therefore, the present study used ox-LDL to induce an AS model.

\section{Objectives}

The present study aimed to investigate the effects of GAA on an in vitro AS model as well as its underlying mechanism, which may provide theoretical guidance for the clinical application of GAA in the treatment of AS.

\section{Materials and methods}

\section{Cell culture and treatments}

Human monocytes (THP-1) were purchased from the American Type Culture Collection (ATCC, Manassas, USA) and cultured in RPMI 1640 medium (Sigma-Aldrich, St. Louis, USA) containing 10\% fetal bovine serum (FBS) and antibiotics $(100 \mathrm{U} / \mathrm{mL}$ of penicillin A and streptomycin) at $37^{\circ} \mathrm{C}$ in a humidified atmosphere containing $5 \%$ $\mathrm{CO}_{2}$. A total of $1 \times 10^{5} \mathrm{THP}-1$ cells were collected and cultivated with $100 \mathrm{nM}$ phorbol-12-myristate13-acetate (PMA; Sigma-Aldrich) for $48 \mathrm{~h}$ to stimulate differentiation into macrophages. The harvested macrophages were subsequently cultured in serum-free medium containing ox-LDL (100 ng/mL; Sigma-Aldrich) for $24 \mathrm{~h}$ to induce foam cell formation. The GAA was purchased from YuanYe Biotechnology Co., Ltd (Shanghai, China). The cells were then treated with various concentrations of GAA $(20 \mu \mathrm{m}$, $50 \mu \mathrm{m}$ or $70 \mu \mathrm{m}$ ) or co-treated with a PPAR $\gamma$ activator. The transfection of THP-1 cells with the overexpression plasmid of Notch1 was performed using Lipofectamine 2000 (Invitrogen, Carlsbad, USA).

\section{CCK-8}

A total of $2 \times 10^{4}$ cells were plated into 96-well plates. After the indicated treatment, the Cell Counting Kit-8 (CCK-8; Glpbio, Montclair, USA) reagent was mixed in the culture medium and added to the 96-well plates. These cells were then incubated for $1 \mathrm{~h}$. Next, the absorbance of the cells was measured using a spectrophotometer (Thermo Fisher Scientific, Waltham, USA). The absorbance at $450 \mathrm{~nm}$ was measured with a microplate reader (Thermo Fisher Scientific).

\section{ELISA}

The levels of tumor necrosis factor- $\alpha$ (TNF- $\alpha)$, IL- $1 \beta$ and IL- 6 in the macrophages were evaluated using commercial enzyme-linked immunosorbent assay (ELISA) kits according to the manufacturer's recommendations (Abcam, Cambridge, UK).

\section{Western blot}

Total proteins were extracted from TPC-1 cells with RIPA buffer (Beyotime, Shanghai, China) and the protein concentration was measured using the BCA Protein Assay Kit (Merck Millipore, Burlington, USA). Next, $40 \mu \mathrm{g}$ of protein in each group was separated using sodium dodecyl sulfate polyacrylamide gel electrophoresis (SDS-PAGE) and transferred to polyvinylidene fluoride (PVDF) membranes (Merck Millipore). The membranes were blocked with $5 \%$ skimmed milk. These blots were then incubated with diluted primary antibodies for the target proteins at $4^{\circ} \mathrm{C}$ overnight, and then secondary antibodies (Santa Cruz 
Biotechnology, Santa Cruz, USA) were added to the membranes and incubated. Image software (National Institutes of Health, Bethesda, USA) was used to determine the intensities. The expression of protein was normalized to GAPDH levels to correct for loading.

\section{Detection of reactive oxygen species and malondialdehyde}

The detection of reactive oxygen species (ROS) was conducted using a commercially available ROS kit (Jiancheng Bioengineering Institute, Shanghai, China), while the detection of malondialdehyde (MDA) was conducted using the corresponding MDA kit from Jiancheng Bioengineering Institute (Nanjing, China) in line with the manufacturer's instructions.

\section{Oil red $\mathrm{O}$ staining of macrophages}

The treated macrophages were collected, fixed in $10 \%$ formaldehyde solution for $20 \mathrm{~min}$, washed with phosphatebuffered saline (PBS) and stained using oil red O (Amresco, Solon, USA) solution (in 60\% isopropanol) for $10 \mathrm{~min}$. Afterwards, the stained macrophages were counterstained with hematoxylin for $3 \mathrm{~min}$. The lipid-stained areas of the slides and cross-sections were observed and photographed using an inverted microscope (Olympus Corp., Tokyo, Japan) at a $\times 200$ magnification. With the application of Image-Pro Plus image analysis software v. 6.0 (Media Cybernetics Inc., Rockville, USA), the lipid droplet content was analyzed.

\section{Measurement of total cholesterol}

Total cholesterol (TC) levels were assayed using the Amplex Red Cholesterol Assay Kit (Invitrogen) according to the manufacturer's protocols.

\section{RT-qPCR}

Trizol reagent (Invitrogen) was used to separate the total RNA from THP-1 cells according to the manufacturer's specifications. Before the real-time quantitative polymerase chain reaction (RT-qPCR) was performed for gene amplification using the SYBR Premix Ex TaqTM GC (TaKaRa, Tokyo, Japan) protocol on a 7900 real-time PCR system (Applied Biosystems, Foster City, USA), the complementary DNA (cDNA) was synthesized from quantified RNA with the use of a PrimeScript TM RT reagent Kit. Relative gene expression was measured using the $2^{-\Delta \Delta C t}$ method. The ratio for the mRNA of interest was normalized to GAPDH. The primers used in this study are as follows: LOX-1 forward: 5'-TTGCCTGGGATTAGTAGTGACC-3'. reverse: 5'- GCTTGCTCTTGTGTTAGGAGGT-3; SR-A forward: 5'-GCAGTGGGATCACTTTCACAA-3', reverse: 5'-AGCTGTCATTGAGCGAGCATC-3'; Notch1 forward 5'-GAGGCGTGGCAGACTATGC-3': reverse 5'-CTTGTACTCCGTCAGCGTGA-3'; PPAR $\gamma$ forward: 5'-GGGATCAGCTCCGTGGATCT-3' reverse: 5'-TGCACTTTGGTACTCTTGAAGTT-3'; CD36 forward: 5'-GGCTGTGACCGGAACTGTG-3', and reverse: 5'AGGTCTCCAACTGGCATTAGAA-3'.

\section{Statistical analyses}

The results were analyzed using GraphPad Prism software v. 5.01 (GraphPad Software, San Diego, USA). All data are presented as the mean \pm standard deviation (SD). Oneway analysis of variance (ANOVA) was utilized to compare differences among multiple groups, followed by Tukey's post hoc test for comparison between 2 groups. Value of $\mathrm{p}<0.05$ was considered to be statistically significant.

\section{Results}

\section{GAA suppresses ox-LDL-induced inflammation and oxidative stress in THP-1 cells}

To confirm the effects of GAA on THP-1 cells induced by ox-LDL, the toxicity of GAA to macrophages was first assessed. The THP-1 cells were treated with different concentrations of GAA $(10 \mu \mathrm{M}, 20 \mu \mathrm{M}, 50 \mu \mathrm{M}$, and $70 \mu \mathrm{M})$ for $24 \mathrm{~h}$. The analysis showed that GAA had no toxic effect on macrophages (Fig. 1A). After $100 \mathrm{nM}$ of PMA treatment of THP-1 cells for $48 \mathrm{~h}$, THP-1 cells were induced to differentiate into macrophages. Next, the macrophages were subjected to ox-LDL stimulation for $24 \mathrm{~h}$. As shown in Fig. 1B, inflammation was induced by ox-LDL through significant elevation of the levels of TNF- $\alpha$, IL-1 $\beta$, IL- 6 , and NF-kB p 65 protein. However, GAA decreased their levels in a dose-dependent manner (Fig. 1C). The high expression of ROS and MDA triggered by ox-LDL was gradually inhibited by increasing the dose of GAA, demonstrating that the possible alteration of oxidative stress status induced by ox-LDL in THP-1 cells was alleviated by increasing the dose of GAA (Fig. 1D). Taken together, these findings show that GAA suppresses ox-LDL-induced inflammation and oxidative stress in THP-1 cells.

\section{GAA suppresses lipid deposition in ox-LDL-induced THP-1 cells}

Abnormal lipid metabolism is an important risk factor for the development of AS. ${ }^{12}$ Therefore, the level of lipid accumulation was measured with oil red $\mathrm{O}$ staining. Compared with the control group, ox-LDL-induced THP-1 cells exhibited markedly higher levels of lipid deposition, which were gradually relieved by GAA treatment (Fig. 2A). Meanwhile, the TC level of THP-1 cells was markedly elevated by ox-LDL. Nevertheless, as the dose of GAA increased, the TC level of THP-1 cells treated by ox-LDL decreased 
A
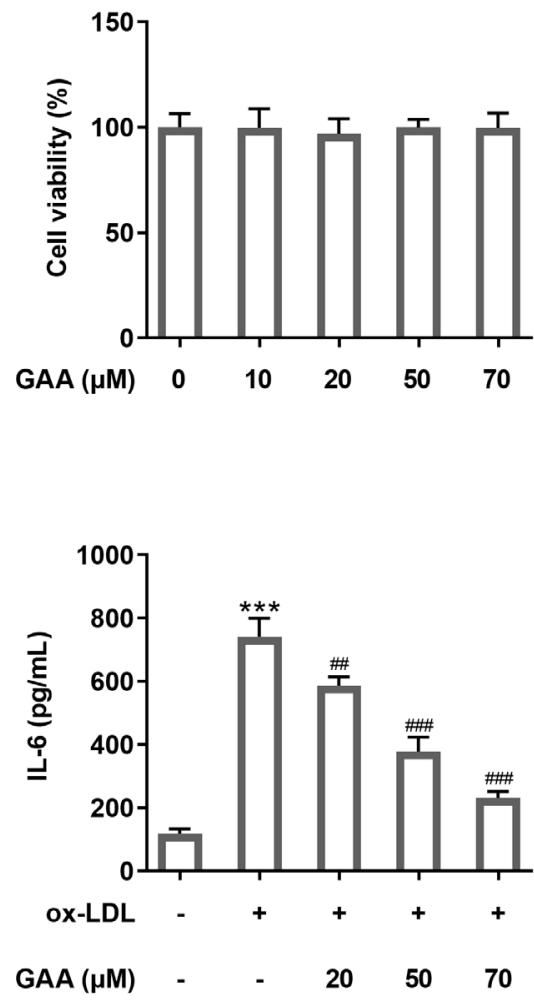

B

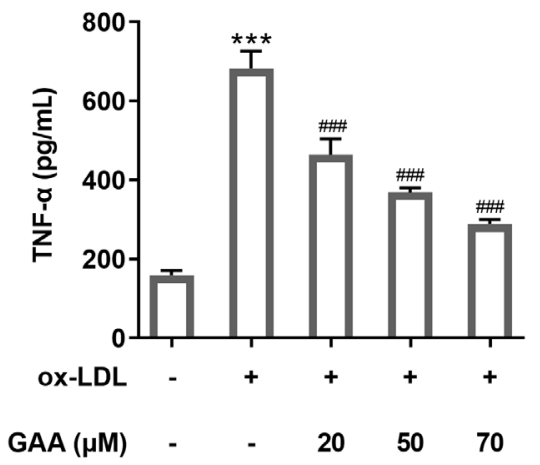

C

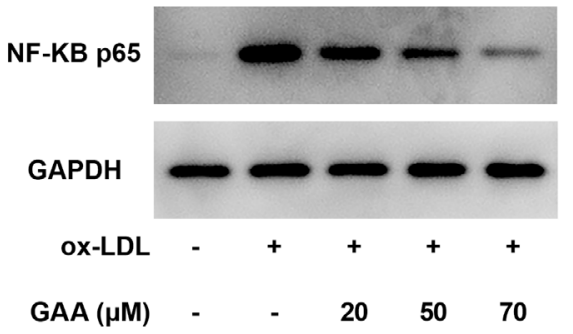

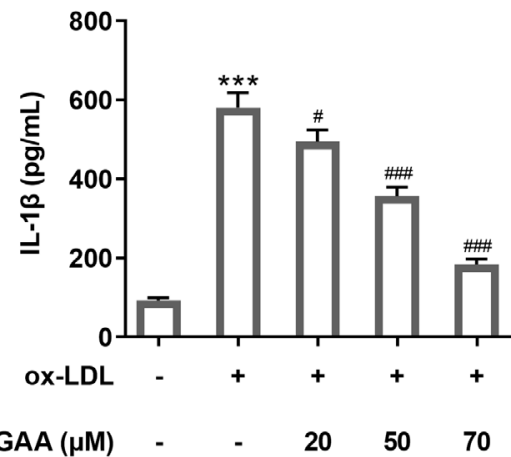

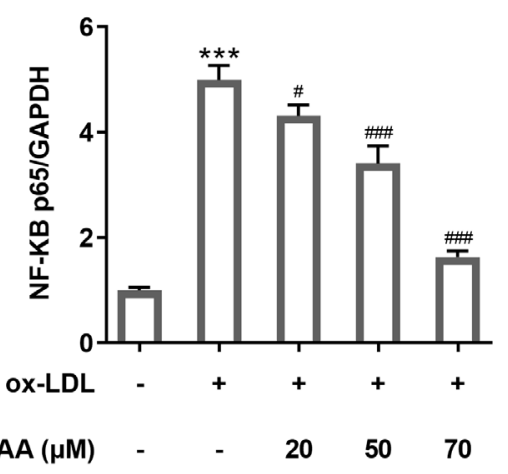

D

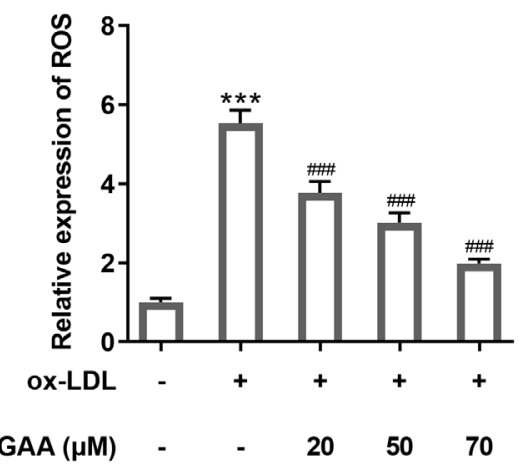

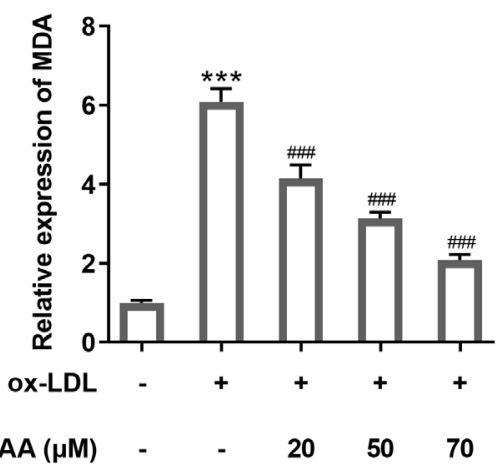

Fig. 1. The effect of GAA on the viability of THP-1 cells. A. The viability of THP-1 cells treated with GAA was assessed with the CCK-8 assay; B. The inflammatory cytokines in ox-LDL-induced THP-1 cells were detected with ELISA; C. The expression level of NF-kB p65 was measured with western blot; D. Oxidative stress-related factors were measured using the corresponding kits. Experimental data are presented as mean \pm SD

*** $p<0.001$ compared to ox-LDL (-), \# $p<0.05$, \#\# $p<0.01$, \#\# $p<0.001$ compared to ox-LDL (+), GAA [ $\mu$ M] (-). ox-LDL - oxidized low-density lipoprotein; ELISA - enzyme-linked immunosorbent assay; SD - standard deviation; CCK-8 - Cell Counting Kit-8; GAA - ganoderic acid A; THP-1 - human monocyte.

(Fig. 2B). The scavenger receptors that play an important part in lipid uptake have a close relationship with the pathogenesis of AS. ${ }^{13}$ Various scavenger receptors (SR) like SR class A (SR-A), SR class B (CD36) and lectin-like oxidized LDL 1 receptor (LOX-1) can recognize and bind to modified LDL. The uptake of modified lipoproteins by these SRs makes great contributions to foam cell formation. ${ }^{14}$ Therefore, RT-qPCR and western blot were adopted to measure the expression of SR-A and CD36. The reduced expression of SR-A and CD36 in ox-LDL-induced THP-1 cells treated with GAA implied the inhibitory role of GAA in lipid uptake (Fig. 3A,B). Thus, the conclusion can be drawn that GAA suppresses lipid deposition in ox-LDL-induced THP-1 cells.

\section{GAA suppresses expression of Notch1/PPARY/CD36 signaling in ox-LDL-induced THP-1 cells}

The effects of GAA on the expression of Notch1, PPAR $\gamma$ and CD36 in ox-LDL-induced THP-1 cells were measured. As seen in Fig. 4, compared with the control group, the expression of Notch1, PPAR $\gamma$ and CD36 increased in the ox-LDL group. Notably, the levels of Notch1, PPAR $\gamma$ and CD36 in ox-LDL-induced THP-1 cells were reduced by GAA. Thus, GAA suppresses the expression of Notch1/PPAR $\gamma / C D 36$ in ox-LDL-induced THP-1 cells. 
A
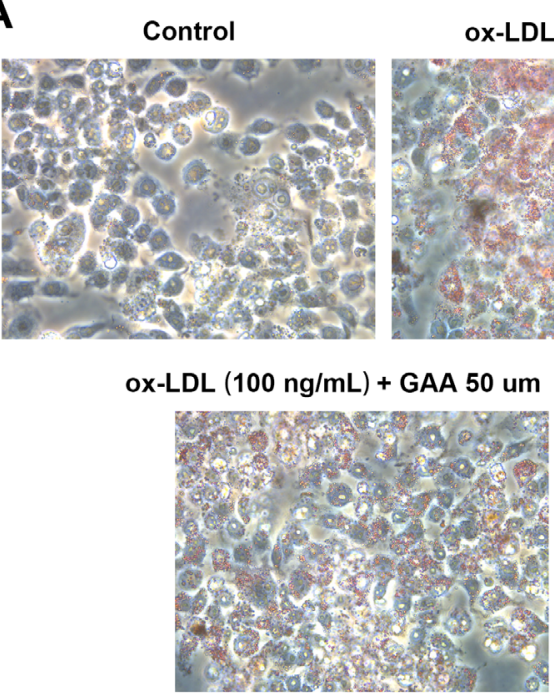

ox-LDL (100 ng/mL)

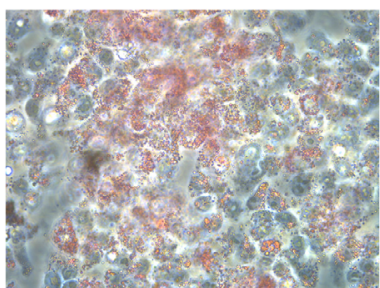

ox-LDL (100 ng/mL) + GAA 20 um

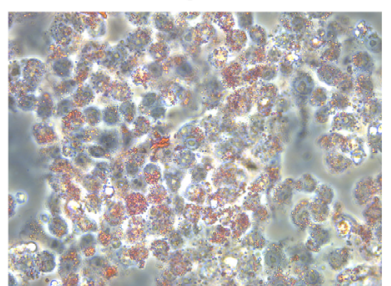

ox-LDL (100 ng/mL) + GAA 70 um

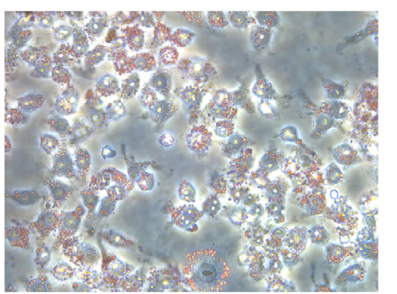

B

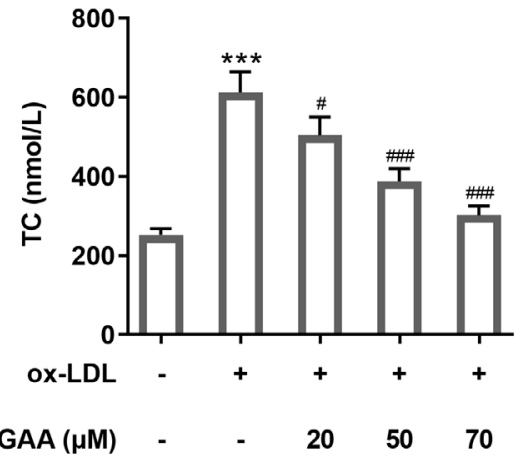

Fig. 2. GAA suppresses lipid deposition in ox-LDL-induced THP-1 cells. A. The level of lipid deposition was detected with oil red O staining; B. The TC level was assayed using the Amplex Red Cholesterol Assay Kit. Values are expressed as mean \pm SD

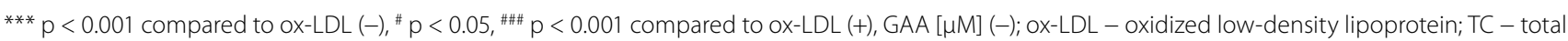
cholesterol; SD - standard deviation; GAA - ganoderic acid A; THP-1 - human monocyte.

A

B

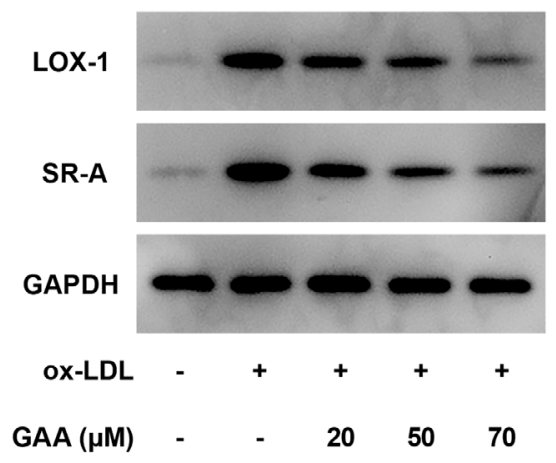

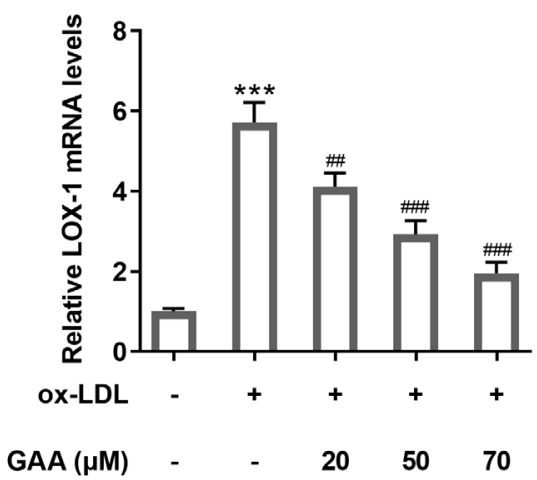

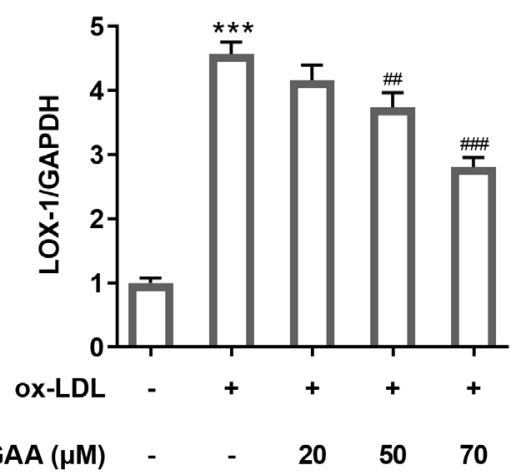

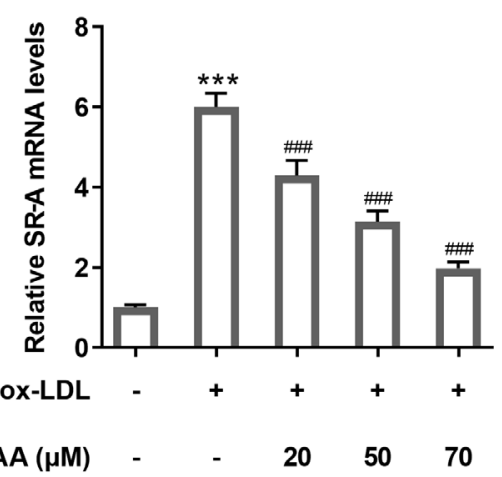

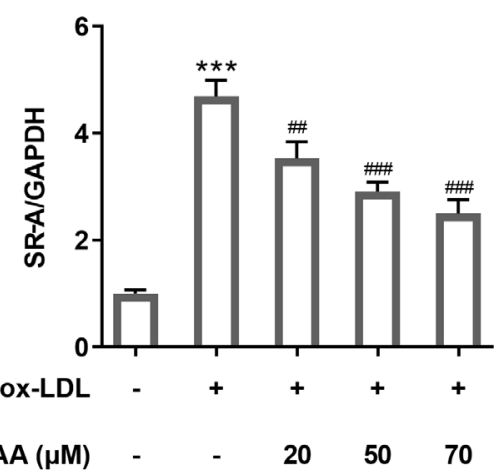

Fig. 3. The expression of scavenger receptors was measured with $\mathrm{qPCR}$ and western blot. Experimental data are shown as mean $\pm \mathrm{SD}$

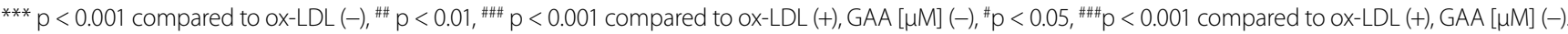
ox-LDL - oxidized low-density lipoprotein; SD - standard deviation; GAA - ganoderic acid A; THP-1 - human monocyte; qPCR - quantitative polymerase chain reaction.

\section{GAA suppresses inflammation and} oxidative stress in ox-LDL-induced THP-1 cells via Notch1/PPARy/CD36 signaling

Next, the expression of Notch1 was increased to a relatively high level to allow detection of its downstream effectors
(Fig. 5A). The overexpression of Notch1 increased the expression of PPAR $\gamma$ and CD36, indicating a positive relationship between Notch1 and its downstream effectors (Fig. 5B). Compared with ox-LDL-induced THP-1 cells with GAA treatment, the expression of inflammatory cytokines and NF-KB p65 was higher in ox-LDL-induced THP-1 cells with 
A

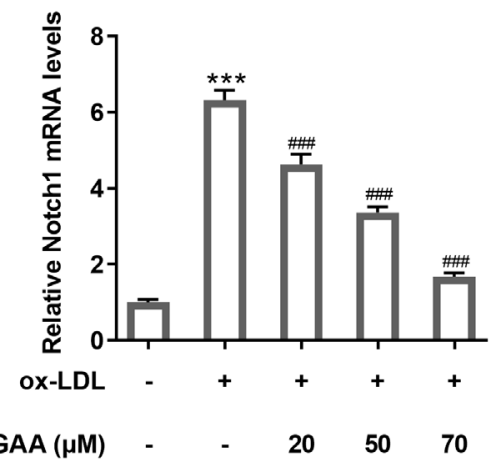

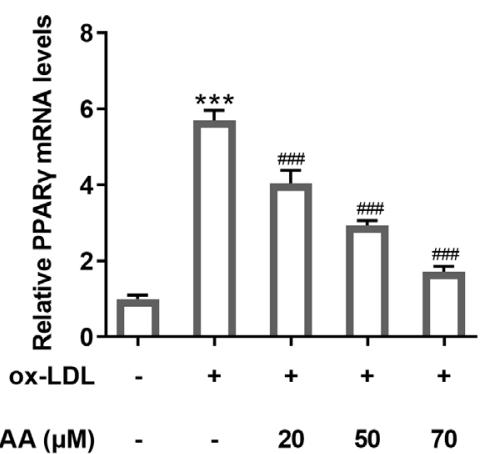

B
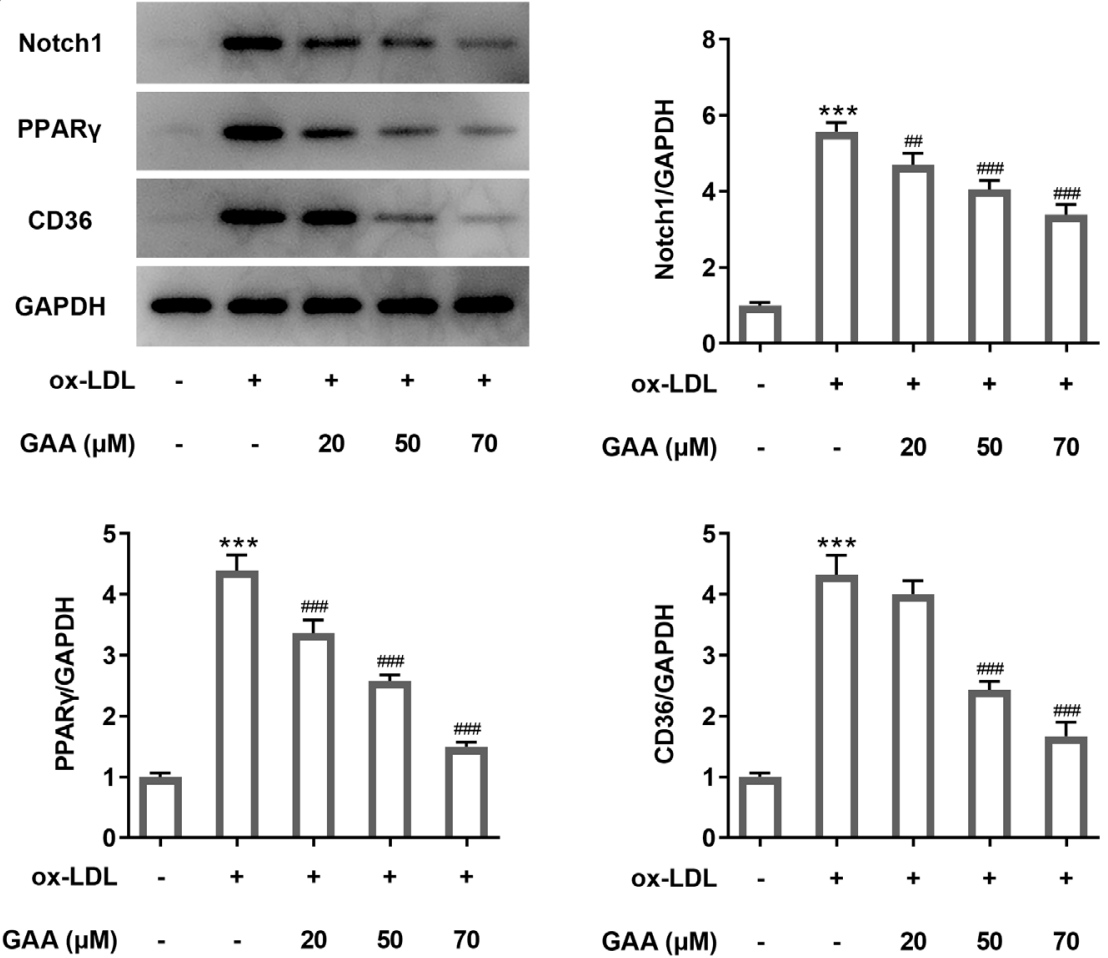

Fig. 4. GAA suppresses the expression of Notch1/PPARY/CD36 in ox-LDL-induced THP-1 cells. A and B. Gene and protein expression in the Notch1/PPARY/ CD36 signaling pathway was assayed using qPCR and western blot, respectively. Experimental data are shown as mean \pm SD

*** $\mathrm{p}<0.001$ compared to ox-LDL (-), $\mathrm{p}<<0.05,{ }^{\# \#} \mathrm{p}<0.01$, ${ }^{\# \#} \mathrm{p}<0.001$ compared to ox-LDL (+) + GAA [ $\mu$ M] (-). ox-LDL - oxidized low-density lipoprotein; SD - standard deviation; GAA - ganoderic acid A; THP-1 - human monocyte; qPCR - quantitative polymerase chain reaction.

combined GAA and the overexpression plasmid of Notch1 (Ov-Notch1) treatment (Fig. 6A,B). Similarly, pretreatment with troglitazone $(10 \mu \mathrm{M})$, which is a PPAR $\gamma$ activator, in oxLDL-induced THP-1 cells upon GAA challenge for $2 \mathrm{~h}$ led to an increased level of inflammatory cytokines. Treatment with Ov-Notch1 and PPAR $\gamma$ activator resulted in higher levels of ROS and MDA, respectively, in ox-LDL-induced THP-1 cells with GAA treatment (Fig. 6C). Therefore, GAA suppresses inflammation and oxidative stress in ox-LDL-induced THP-1 cells via Notch1/PPAR $\gamma / C D 36$ signaling.

\section{GAA inhibits lipid deposition} in ox-LDL-induced THP-1 cells via Notch1/PPAR $\gamma / C D 36$ signaling

To further confirm the role of the Notch1/PPAR $\gamma / \mathrm{CD} 36$ signal in lipid deposition in the AS model, the level of lipid accumulation was detected in ox-LDL-induced THP-1 cells with different treatments. As Fig. 7A shows, lipid accumulation reached the highest level in ox-LDL-induced THP-1 cells without any other treatment. The GAA greatly relieved lipid accumulation in ox-LDL-induced THP-1 cells. Furthermore, the addition of Ov-Notch1 or PPAR $\gamma$ activator promoted the formation of oil droplets. Notch signaling is recognized to regulate PPARY protein stability in human macrophages. ${ }^{15}$ In our study, the TC content, which 
A
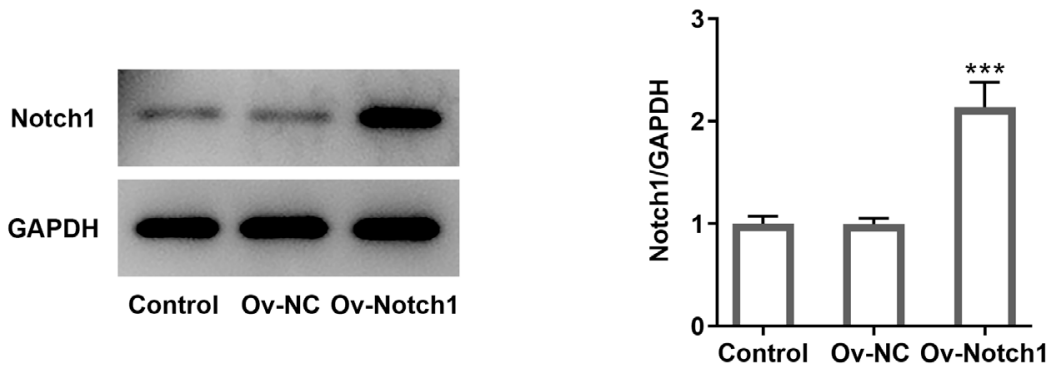

B

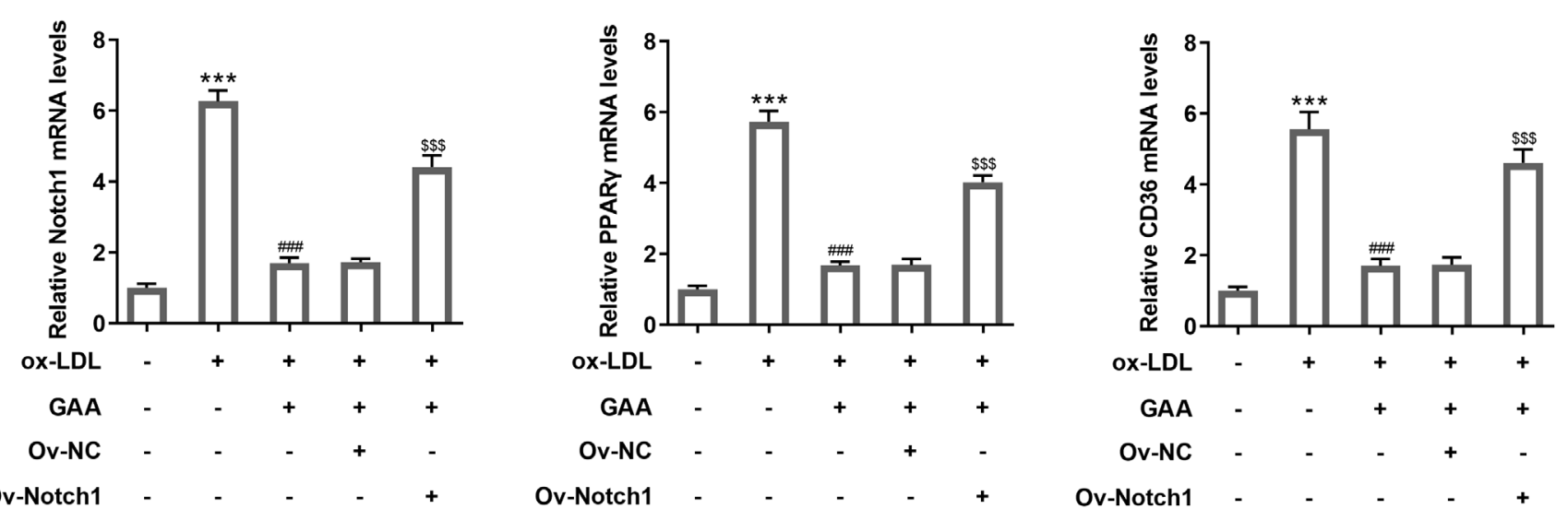

C
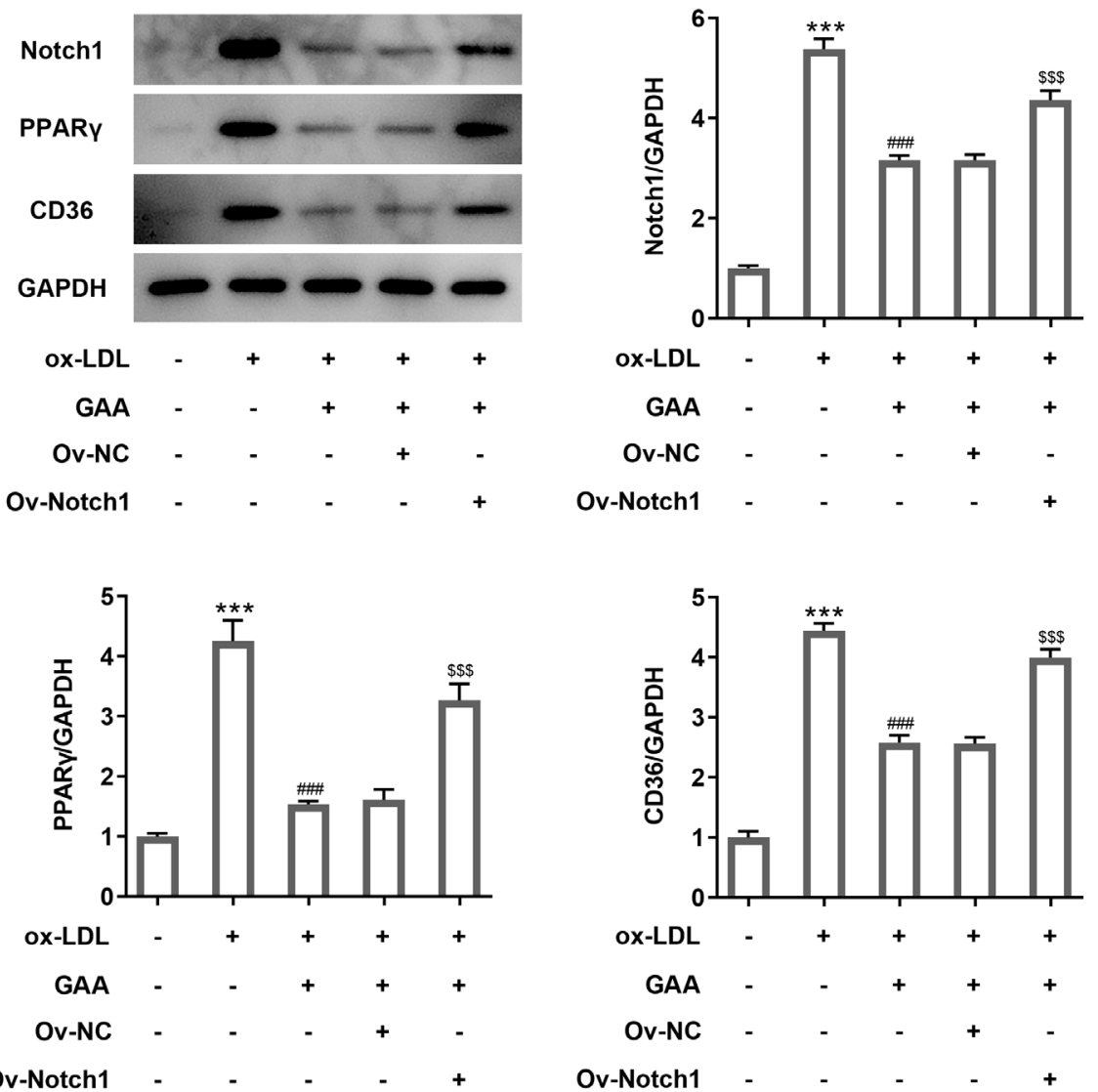

Fig. 5. Notch1 overexpression reversed the effects of GAA. GAA suppresses inflammation and oxidative stress in ox-LDL-induced THP-1 cells via Notch1/ PPARY/CD36 signaling pathway. A. The level of Notch1 was detected after it was overexpressed in THP-1 cells; B. Protein expression in the Notch1/PPARY/ CD36 signaling pathway was assayed using western blot. Values are expressed as mean \pm SD

*** $p<0.001$ compared to Ov-NC, \#\#\# $p<0.001$ compared to ox-LDL (+) + GAA (-) + Ov-NC (-) + Ov-Notch1 (-), \$\$\$ $p<0.001$ compared to ox-LDL (+) + GAA $(+)+$ Ov-NC (+) +Ov-Notch1 (-). ox-LDL - oxidized low-density lipoprotein; SD - standard deviation; GAA - ganoderic acid A; THP-1 - human monocyte. 
A
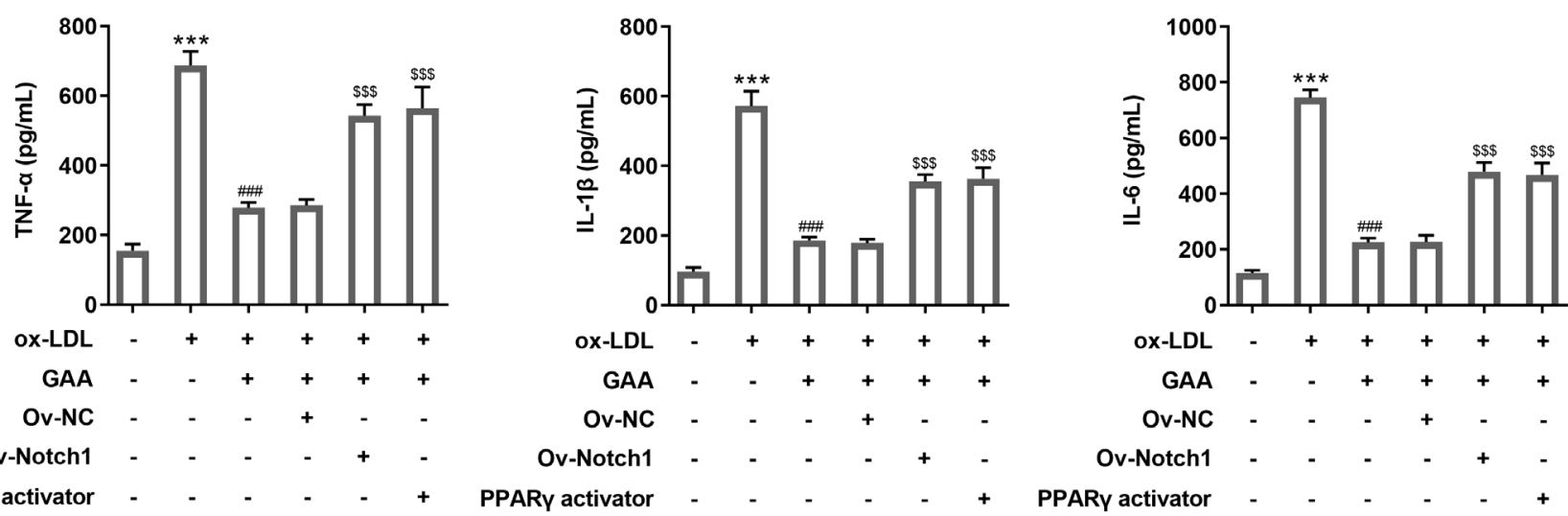

B
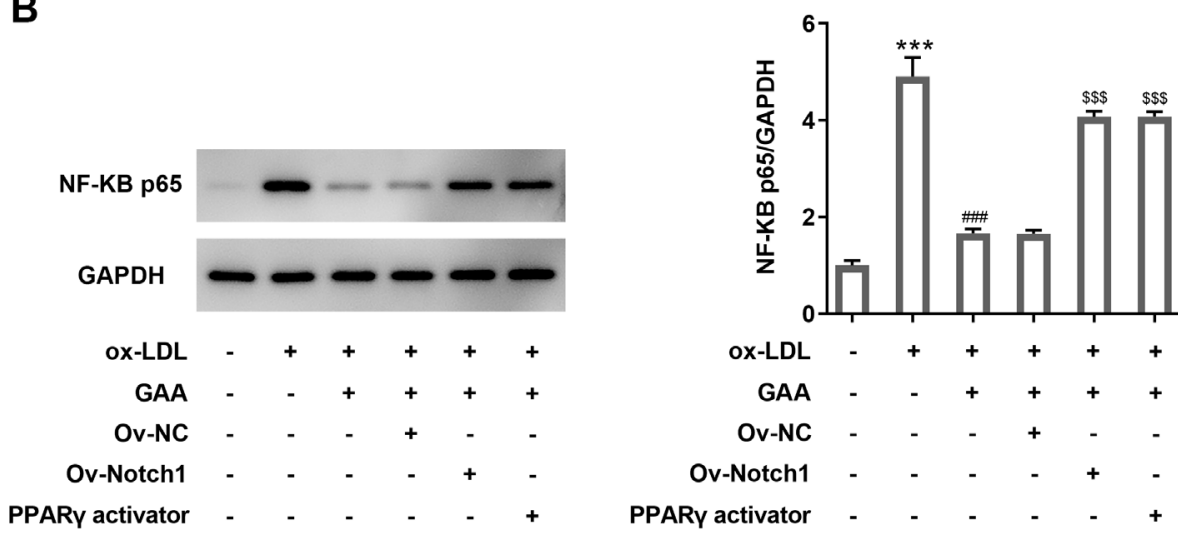

C
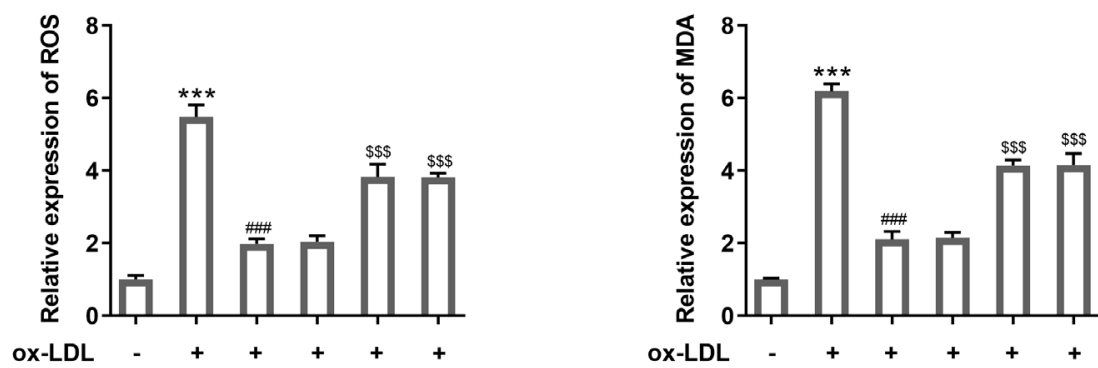

ox-LDL

GAA

Ov-NC

Ov-NC

Ov-Notch1

PPARY activator

PPARy activator

Fig. 6. GAA suppresses inflammation and oxidative stress in ox-LDL-induced THP-1 cells via Notch1/PPARY/CD36 signaling pathway. A. The inflammatory cytokines in ox-LDL-induced THP-1 cells were detected with ELISA; B. The expression level of NF-KB p65 was measured using western blot; C. The oxidative stress-related factors were measured using the corresponding kits. Values are expressed as mean \pm SD

*** $\mathrm{p}<0.001$ compared to Ov-NC,\#\# $\mathrm{p}<0.001$ compared to ox-LDL $(+)+\mathrm{GAA}(-)+$ Ov-NC $(-)+$ Ov-Notch1 (-), \$\$\$ $\mathrm{p}<0.001$ compared to ox-LDL (+) + GAA (+) + Ov-NC (+) + Ov-Notch1 (-). ox-LDL - oxidized low-density lipoprotein; SD - standard deviation; ELISA - enzyme-linked immunosorbent assay; GAA - ganoderic acid A; THP-1 - human monocyte; PPARY - peroxisome proliferator-activated receptor gamma; NC - negative control.

was reduced by GAA, increased by Notch1 overexpression or PPAR $\gamma$ activator (Fig. 7B). The changes in the expression levels of LOX-1 and SR-A demonstrate the same alteration as the abovementioned results (Fig. 8A,B). Therefore, the conclusion that GAA inhibits lipid deposition in ox-LDL-induced THP-1 cells via Notch1/PPAR $\gamma /$ CD36 signaling can be drawn. However, Ov-Notch1 and PPAR $\gamma$ activator can partly reverse the suppressive effects of GAA on lipid deposition in ox-LDL-induced THP-1 cells.
Together, these results demonstrate that Notch1/PPAR $\gamma /$ CD36 signaling pathway is involved in the effects of GAA on lipid deposition.

\section{Discussion}

Macrophages play a critical role in innate immunity and diseases of high prevalence, including cancer, obesity 
Fig. 7. GAA inhibits lipid deposition in ox-LDL-induced THP-1 cells via Notch1/PPARY/CD36 signaling pathway. A. The level of lipid deposition was detected with oil red O staining; B. The TC level was assayed using the Amplex Red Cholesterol Assay Kit. Values are expressed as mean \pm SD

*** $\mathrm{p}<0.001$ compared to Ov-NC, \#\#\# $\mathrm{p}<0.001$ compared to ox-LDL $(+)+\mathrm{GAA}(-)+\mathrm{Ov}-\mathrm{NC}(-)+$ Ov-Notch1 (-), \$\$ $\mathrm{p}<0.001$ compared to ox-LDL (+) + GAA (+) + Ov-NC (+) + Ov-Notch1 (-). ox-LDL - oxidized low-density lipoprotein; TC - total cholesterol; SD - standard deviation; GAA - ganoderic acid A; THP-1 - human monocyte; PPARY - peroxisome proliferator-activated receptor gamma; NC - negative control.

A

B

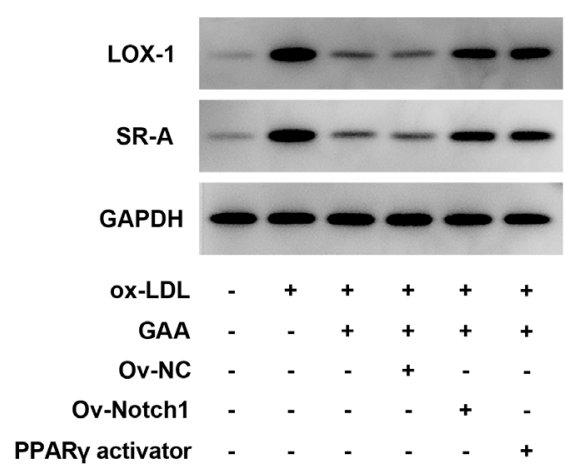

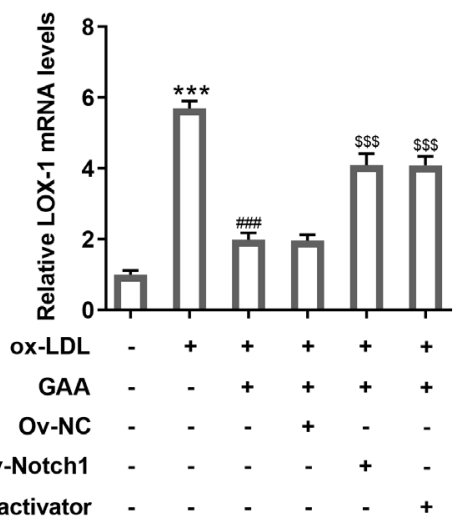

PPARy activator $\quad-\quad-\quad-\quad-\quad+\quad+$

Fig. 8. GAA inhibits the expression of scavenger receptors in ox-LDL-induced THP-1 cells via Notch1/PPARY/CD36 signaling pathway. The expression of scavenger receptors was measured with qPCR and western blot. Values are expressed as mean $\pm S D$

*** $\mathrm{p}<0.001$ compared to Ov-NC, ${ }^{* \# \#} \mathrm{p}<0.001$ compared to ox-LDL (+) + GAA (-) + Ov-NC (-) + Ov-Notch1 (-), $\$ \$ \$ p<0.001$ compared to ox-LDL (+) + GAA (+) + Ov-NC (+) + Ov-Notch1 (-). ox-LDL - oxidized low-density lipoprotein; TC - total cholesterol; SD - standard deviation; GAA - ganoderic acid A; THP-1 - human monocyte; QPCR - quantitative polymerase chain reaction; PPARY - peroxisome proliferator-activated receptor gamma; NC - negative control. 
and AS. ${ }^{16}$ When monocytes arrive at an injury site, they transform into macrophages, the activation of which can respond to signals in damaged tissues. ${ }^{17}$ Macrophages are thought to play a pathogenic role in the formation of atherosclerotic plaques. The roles of macrophages in AS are tied to its polarization. Polarized macrophages are divided into 2 phenotypes: M1 and M2. ${ }^{2}$ Activated M1 macrophages can be induced by lipopolysaccharides (LPS), while M2 macrophages are activated after exposure to various cytokines, including IL-4, IL-13 and IL-10.

It was previously reported that pro-inflammatory factors, including IL-1 $\beta$, IL- 6 and TNF- $\alpha$, are critical contributors to the progression of AS. Thus, it is of great value to find novel targets for the suppression of inflammation related to AS lesions. ${ }^{3}$ Together with the decrease in the level of NF-KB p65, which is a transcription factor involved in the regulation of inflammation, the inhibition of these cytokines by GAA in THP-1 cells under ox-LDL conditions potently implies the role of GAA in blocking the inflammatory response ${ }^{18}$ Consistent with our results, a previous report suggested that GAA caused decreased levels of IL-1 $\beta$, IL- 6 and TNF- $\alpha$ in mice with lung injury. ${ }^{7}$ Anti-oxidant therapy is considered an effective method for the prevention and treatment of AS because the inflammatory changes triggering the occurrence of AS can be inhibited by suppressing oxidized lipoprotein and lipid formation and oxidative stress. ${ }^{19}$ Due to the remarkable effects of GAA on alleviating inflammation in multiple injuries and blocking the development of cancers, GAA was hypothesized to play a critical role in AS. We identified that GAA was able to suppress the release of inflammatory factors and oxidative stress in ox-LDL-induced THP-1 cells. Furthermore, lipid deposition induced by ox-LDL in THP-1 cells was relieved by GAA.

Notch signaling is a critical transcriptional factor in the regulation of differentiation and proliferation of stem cells. ${ }^{20}$ The Notch signaling pathway is involved in interactions among cells throughout the organs of the body in a large number of diseases. It is suggested that the cellular processes of monocytes and the modulation of macrophages are determined by this signaling pathway. ${ }^{21,22}$ In the present study, Notch signaling was induced by ox-LDL but inhibited by GAA. Furthermore, the overexpression of Notch1 relieved the inhibitory effect of GAA on the reduction of inflammatory factors and oxidative stress. PPAR $\gamma$, a ligand-activated transcription factor that regulates fatty acid metabolism, plays a role in lipid deposition, cell differentiation and inflammation. ${ }^{23}$ It has been reported that ox-LDL can strongly activate PPAR $\gamma$ to regulate gene expression in macrophages. ${ }^{24}$ The expression of PPAR $\gamma$ was markedly increased after ox-LDL was used to induce THP-1 cells, suggesting successful activation of PPAR $\gamma$ by ox-LDL. The GAA treatment potently inhibited its expression, while the PPAR $\gamma$ activator, which activates the PPAR $\gamma$ signal, led to further inflammation and oxidative stress. Therefore, the mechanism by which
GAA alleviates inflammation and oxidative stress might be related to a PPAR $\gamma$-dependent signal. Internalized ox-LDL supplies rich oxidized fatty acids that serve as ligands to PPAR $\gamma$, thereby inducing genes such as CD36. ${ }^{25}$ The role of CD36 in long-chain fatty acid uptake and oxidation has been deeply studied. The findings have shown that it participates in processes like apoptosis, inflammation and $\mathrm{AS} .{ }^{26} \mathrm{CD} 36$ has great potential in scavenging ox-LDL to protect intima from cholesterol deposits and atherosclerotic lesions. ${ }^{27}$ Knockout of CD36 is associated with enhanced symptoms in patients with atherosclerotic cardiovascular diseases. ${ }^{28}$ In this study, GAA inhibited the expression of Notch1, PPAR $\gamma$ and CD36 as well as lipid deposition. The expression of scavenger receptors could also be inhibited by GAA.

\section{Limitations}

There is still a lack of in vivo evidence to confirm the role and action mechanism of GAA in AS. Furthermore, how GAA affects Notch1/PPAR $\gamma / C D 36$ signaling requires deeper investigation.

\section{Conclusions}

Ganoderic acid A inhibits ox-LDL-induced THP-1-derived macrophage inflammation and lipid deposition via Notch1/PPAR $\gamma / C D 36$ signaling, which may provide theoretical guidance for the clinical application of GAA in AS treatment.

The datasets used and/or analyzed in the current study are available from the corresponding author on reasonable request.

\section{ORCID iDs}

Tao Wang (1) https://orcid.org/0000-0001-6355-392X

Huihe Lu (i) https://orcid.org/0000-0003-1474-8012

\section{References}

1. Wang R, Nascimento BR, Neuenschwander FC. Atherosclerosis and inflammation: Still a long way to go. Arq Bras Cardiol. 2020;114(4): 699-700. doi:10.36660/abc.20200219

2. Moore KJ, Tabas I. Macrophages in the pathogenesis of atherosclerosis. Cell. 2011;145(3):341-355. doi:10.1016/j.cell.2011.04.005

3. Liu $\mathrm{P}, \mathrm{Xu} \mathrm{Y}, \mathrm{Yan} \mathrm{H}$, et al. Characterization of molecular signature of the roots of Paeonia lactiflora during growth. Chin J Nat Med. 2017; 15(10):785-793. doi:10.1016/S1875-5364(17)30110-3

4. Wang X, Sun D, Tai J, et al. Ganoderic acid A inhibits proliferation and invasion, and promotes apoptosis in human hepatocellular carcinoma cells. Mol Med Rep. 2017;16(4):3894-3900. doi:10.3892/mmr. 2017.7048

5. Jiang J, Grieb B, Thyagarajan A, et al. Ganoderic acids suppress growth and invasive behavior of breast cancer cells by modulating AP-1 and NF-kappaB signaling. Int J Mol Med. 2008;21(5):577-584. PMID:18425349

6. Liu F, Shi K, Dong J, et al. Ganoderic acid A attenuates high-fat-dietinduced liver injury in rats by regulating the lipid oxidation and liver inflammation. Arch Pharm Res. 2020;43(7):744-754. doi:10.1007/ s12272-020-01256-9 
7. Wan B, Li Y, Sun S, et al. Ganoderic acid A attenuates lipopolysaccharide-induced lung injury in mice. Biosci Rep. 2019;39(5):BSR20190301. doi:10.1042/BSR20190301

8. Gill BS, Navgeet, Kumar S. Antioxidant potential of ganoderic acid in Notch-1 protein in neuroblastoma. Mol Cell Biochem. 2019;456(1-2): 1-14. doi:10.1007/s11010-018-3485-7

9. Ernst IM, Wagner $A E$, Lipinski $S$, et al. Cellular uptake, stability, visu

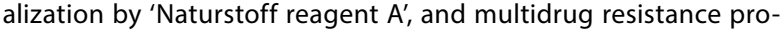
tein 1 gene-regulatory activity of cyanidin in human keratinocytes. Pharmacol Res. 2010;61(3):253-258. doi:10.1016/j.phrs.2009.10.006

10. Liu D, Wang X, Zhang M, et al. WISP1 alleviates lipid deposition in macrophages via the PPARgamma/CD36 pathway in the plaque formation of atherosclerosis. J Cell Mol Med. 2020;24(20):11729-11741. doi:10.1111/jcmm.15783

11. Chistiakov DA, Melnichenko AA, Grechko AV, et al. Potential of antiinflammatory agents for treatment of atherosclerosis. Exp Mol Pathol. 2018;104(2):114-124. doi:10.1016/j.yexmp.2018.01.008

12. Tian M, Zhao J, Mi X, et al. Progress in research on effect of PM2.5 on occurrence and development of atherosclerosis. J Appl Toxicol. 2020;41(4). doi:10.1002/jat.4110

13. Moore KJ, Kunjathoor VV, Koehn SL, et al. Loss of receptor-mediated lipid uptake via scavenger receptor A or CD36 pathways does not ameliorate atherosclerosis in hyperlipidemic mice. J Clin Invest. 2005;115(8):2192-2201. doi:10.1172/JCI24061

14. Kelley JL, Ozment TR, Li C, et al. Scavenger receptor-A (CD204): A two-edged sword in health and disease. Crit Rev Immunol. 2014;34(3): 241-261. doi:10.1615/critrevimmunol.2014010267

15. Sangphech N, Keawvilai P, Palaga T. Notch signaling increases PPARY protein stability and enhances lipid uptake through AKT in IL-4-stimulated THP-1 and primary human macrophages. FEBS Open Bio. 2020;10(6):1082-1095. doi:10.1002/2211-5463.12858

16. Mahley RW. Cellular and molecular biology of lipoprotein metabolism in atherosclerosis. Diabetes. 1981;30(Suppl 2):60-65. doi:10.2337/ diab.30.2.s60

17. Brown BN, Ratner BD, Goodman SB, et al. Macrophage polarization: An opportunity for improved outcomes in biomaterials and regenerative medicine. Biomaterials. 2012;33(15):3792-3802. doi:10.1016/j. biomaterials.2012.02.034
18. Berliner JA, Navab M, Fogelman AM, et al. Atherosclerosis: Basic mechanisms. Oxidation, inflammation, and genetics. Circulation. 1995;91(9):2488-2496. doi:10.1161/01.cir.91.9.2488

19. Witztum JL, Steinberg D. The oxidative modification hypothesis of atherosclerosis: Does it hold for humans? Trends Cardiovasc Med. 2001;11(3-4):93-102. doi:10.1016/s1050-1738(01)00111-6

20. Huang $Y$, Yang $X, W u$, et al. Gamma-secretase inhibitor induces adipogenesis of adipose-derived stem cells by regulation of Notch and PPAR-gamma. Cell Prolif. 2010;43(2):147-156. doi:10.1111/j.13652184.2009.00661.x

21. Hu X, Chung AY, Wu I, et al. Integrated regulation of Toll-like receptor responses by Notch and interferon-gamma pathways. Immunity. 2008;29(5):691-703. doi:10.1016/j.immuni.2008.08.016

22. Jiang $S, D a Y, H a n S$, et al. Notch ligand Delta-like1 enhances degranulation and cytokine production through a novel Notch/Dok-1/ MAPKs pathway in vitro. Immunol Res. 2018;66(1):87-96. doi:10.1007/ s12026-017-8977-0

23. Lee KJ, Kim HA, Kim PH, et al. Ox-LDL suppresses PMA-induced MMP-9 expression and activity through CD36-mediated activation of PPARgamma. Exp MolMed. 2004;36(6):534-544. doi:10.1038/emm.2004.68

24. Nagy L, Tontonoz P, Alvarez JG, et al. Oxidized LDL regulates macrophage gene expression through ligand activation of PPARgamma. Cell. 1998;93(2):229-240. doi:10.1016/s0092-8674(00)81574-3

25. Chawla $A$, Boisvert WA, Lee $\mathrm{CH}$, et al. A PPAR gamma-LXR-ABCA1 pathway in macrophages is involved in cholesterol efflux and atherogenesis. Mol Cell. 2001;7(1):161-171. doi:10.1016/s1097-2765(01)00164-2

26. Febbraio M, Hajjar DP,Silverstein RL. CD36: A class B scavenger receptor involved in angiogenesis, atherosclerosis, inflammation, and lipid metabolism. J Clin Invest. 2001;108(6):785-791. doi:10.1172/JCI14006

27. Febbraio M, Podrez EA, Smith JD, et al. Targeted disruption of the class $B$ scavenger receptor CD36 protects against atherosclerotic lesion development in mice. J Clin Invest. 2000;105(8):1049-1056. doi:10.1172/JCI9259

28. Yuasa-Kawase M, Masuda D, Yamashita T, et al. Patients with CD36 deficiency are associated with enhanced atherosclerotic cardiovascular diseases. J Atheroscler Thromb. 2012;19(3):263-275. doi:10.5551/ jat.10603 